\title{
Applying a Dynamic Method to the Measurement of Ion Mobility
}

\author{
Gökhan Baykut, Oliver von Halem, and Oliver Raether \\ Bruker Daltonik GmbH, Bremen, Germany
}

A dynamic method is applied to measure the mobility of gas-phase ions in the dual ion funnel interface of the electrospray source of a quadrupole orthogonal time-of-flight mass spectrometer. In a new operational mode, a potential barrier was formed in the second ion funnel of the mass spectrometer and then progressively increased. In this region, a flow of gas drags the ions into the mass spectrometer while the electric force applied by the potential barrier decelerates them. Ions with lower mobility can be carried by the gas flow more easily than those with high mobility. Thus, electrical forces can block the more mobile ions more easily. Hence, the electric barrier formed in the ion funnel permits only ions below a certain mobility threshold to enter the mass spectrometer. When the barrier voltage is increased, this threshold moves from high to low mobilities. Ions with mobilities above the threshold cannot enter the mass spectrometer, and their signal decreases to zero. Thus, in a barrier voltage scan, mass spectrometric signals of ions sequentially disappear. Differentiation of these decreasing ion signal curves produces peaks from which an ion mobility spectrum can be reconstructed. Blocking voltages, i.e., the positions of the peaks on the barrier voltage scale are directly related to the mobility of these ions. An internal calibration using ions with known mobility values helps determine the unknown ion mobilities and allows calculation of ionic cross sections. (J Am Soc Mass Spectrom 2009, 20, 2070-2081) (C) 2009 American Society for Mass Spectrometry

I on mobility studies using classic drift tubes to investigate gas-phase ion-molecule reactions began more than 40 years ago in chemical physics laboratories [1-3]. In the last decades of the twentieth century, ion mobility spectrometry with mass spectrometric ion detection (IMS-MS) became increasingly popular among the analytical chemists. In recent years, mass spectrometry of mobility-separated gas-phase ions found numerous applications in fields ranging from small molecule analyses to large cluster and biopolymer studies. The primary driving force behind the contemporary IMS-MS excitement in the scientific community is the possibility of measuring the gas-phase ion mobilities, investigating higher order molecular structures, in particular conformational isomers, as well as physically separating these structural or conformational isomers for further analytical experiments. In mass spectrometry, the cross section-to-charge ratio (shape-to-charge ratio) of ions became important complementary information to their mass-to-charge-ratio [4-12].

Measuring the mobility of an ion helps calculate its ionic cross section, which in turn helps in the estimation of the most likely structure for the conformational isomer. One of the important recent applications of the IMS-MS is the investigation of protein folding in neurodegenerative diseases [13]. Additionally, since homologous series of different compounds usually show

Address reprint requests to Dr. G. Baykut, Bruker Daltonik GmbH, Fahrenheitstrasse 4, 28359 Bremen, Germany. E-mail: gb@bdal.de different structural densities, displaying an $\mathrm{m} / \mathrm{z}$ versus mobility diagram gives a very practical overview of the compound groups being studied, independent of secondary mass spectrometric investigations, e.g., collision induced dissociation (CID). Peptides, for instance, generally have more compact structures compared with lipids of the same mass range. Thus, an $m / z$ versus mobility diagram of a mixture of peptides and lipids upon IMS-MS analysis shows two distinct patterns [10, 11].

In classic ion mobility spectrometry, ions are pulsed into a drift tube (IMS cell) filled with a buffer gas, and accelerated by an electric field. Due to the frictional drag force originating from collisions with the gas molecules, ions drift with constant velocities through the IMS cell tube. This constant velocity $v$, at which the electrical force equals to the frictional force, is proportional to the applied electric field $E$. The proportionality constant $K$ is the ion mobility.

$$
v=K E
$$

Ions with higher mobility (smaller cross section, higher electric charge) arrive earlier at the end of a drift tube than ions with lower mobility (larger cross section, lower electric charge). In IMS-MS systems ions continue their route into the mass spectrometer to be analyzed. Quadrupole mass spectrometers or orthogonalacceleration extraction time-of-flight mass spectrometers are frequently used as mass analyzers. A mobility- 
based separation of a pulsed group of ions in the drift tube leads to a sequential arrival of ions separated by their mobilities. This "arrival time distribution" is an ion mobility spectrum. Longer ion mobility drift tubes $(\geq 2 \mathrm{~m}$ ) achieve mobility resolving powers $\mathrm{K} / \Delta \mathrm{K}$ of 100 or higher [14-16].

Interesting experiments for measuring collision cross sections of ions were also reported by Covey and Douglas, [17], by Cox et al. [18], and by Gill et al. [19], who used tandem quadrupole mass spectrometers. In these experiments, ions traveling through the collision cell lost a certain portion of their kinetic energy depending on their cross sections. Larger ions suffered a more significant loss of energy in the collision cell. They were unable to enter the third quadrupole due to its DCoffset voltage which was set higher than the voltage of the collision cell [17, 18]. This effect was used for checking the translational energy and obtaining information about collision cross section of ions. In the setup used by Gill et al. [19], a stopping voltage was applied to the exit lens of the RF-only hexapole collision cell of the tandem quadrupole. After collisions in the cell only those ions that had sufficient remaining translational energy passed the stopping voltage and produced a mass spectrometric signal. Collision cross sections were determined by studying the decrease of signal intensities while varying the stopping voltage at different collision gas pressures [19].

Some of the ion mobility spectrometers use flowing buffer gas instead of a stationary gas. An example is an IMS-MS system which uses a buffer gas in laminar flow perpendicular to pulsed ions [20]. In this case, the entrance and exit orifices of the ion mobility cell are offset from one another, so that the arrival of ions at the exit orifice depends on ion mobilities and on the applied electric field when the buffer gas flow rate is kept constant.

In our current studies a different method is applied to measure ion mobilities, which we perform in one of the ion funnels of the mass spectrometer. The use of ion funnels began already in late 1990s to increase the ion transmission efficiency between the atmospheric pressure ion sources and the mass spectrometric high vacuum [21]. Ion funnels can be operated at relatively high pressures, e.g., 1-10 mbar and usually consist of an RF ion guide normally made of ring electrodes with decreasing inner diameters and often with a superimposed potential gradient along the ring stack. The use of ion funnels instead of skimmers, which were formerly employed in this interface region, dramatically increased the ion transmission efficiency into the mass spectrometer. Since ion funnels are positioned in the high-pressure regions, the transition of ions is supported by the viscous gas flow from the high-pressure to the low-pressure regions of these mass spectrometric devices. A study of an adjustable low-mass filter reported by Page et al. [22] described a way of eliminating small ions up to $\mathrm{m} / \mathrm{z} 500$ by applying an adjustable potential barrier to the conductance limiting plate of the electrodynamic ion funnel interfacing the electrospray source with the mass spectrometer. Unwanted background species usually originating from low mass ions are prohibited from entering the mass spectrometer. The mechanism for filtering these low mass ions in the ion funnel using a potential barrier is explained by the authors with respect to the ion mobilities [22]: The potential barrier blocks species with low masses, i.e., lower cross sections and higher ion mobilities from exiting the ion source. The authors report that the dependence of the low $\mathrm{m} / \mathrm{z}$ cutoff versus the filter voltage was linear.

We can use a potential barrier in an ion funnel to actually measure the mobility of ions. The repelling electric force applied to an ion by the potential barrier is directed against the dragging force applied by the gas flow. Ions of high mobility, i.e., small cross sections and high number of charges, are controlled more easily by electric forces. However, ions with low mobilities are more susceptible to be dragged off by the gas flow, since their cross section-to-charge ratio is larger. At any level of the scanned voltage, the formed barrier restricts the ions with mobilities above a certain value from entering the mass spectrometer and permits only ions below a certain mobility threshold to enter the mass spectrometer. When the barrier voltage is gradually increased the threshold moves from high to low mobilities. Ions with mobilities above this threshold are stopped from entering the mass spectrometer. Their signals do not appear in the mass spectrum. If an ion's mobility corresponds to the current position of the mobility threshold, its signal shows a decreasing curve at this point. Therefore, a gradual increase of the barrier potential allows the scanning of mobilities of ions introduced into the mass spectrometer. Their mass spectrometric signals disappear in sequence at corresponding barrier voltages.

As the barrier voltage is increased, mass spectrometric signals of ions with different mobilities decrease at different voltages and disappear. Negative derivatives of these decreasing signal plots lead to positive peaks. The position of these peaks on the barrier voltage scale is related to the mobility of the corresponding ions. In the following, we present ion mobility experiments in the second ion funnel of the dual ion funnel interface of an electrospray hybrid quadrupole orthogonal time-offlight mass spectrometer (QTOF) and show that this can be a very useful method for measuring ion mobility.

\section{Experimental}

\section{Materials}

Peptides used in ion mobility experiments, angiotensin I, angiotensin II, bradykinin, fibrinopeptide A, neurotensin, $\left[\mathrm{Arg}^{8}\right]$-vasopressin, [des-Glu $\left.{ }^{1}\right]-\mathrm{LHRH}$, bombesin, melittin, the peptide Gly-Arg-Gly-Asp-Ser, polyethylene glycol 400 (PEG 400), and prostaglandin $\mathrm{E}_{1}$, as well as methanol (used as solvent) were obtained from 
Sigma-Aldrich (Taufkirchen, Germany). Distilled and deionized water (Pestanal) was sourced from HoneywellRiedel-de Haën (Seelze, Germany), and formic acid from Fluka (Sigma-Aldrich, Taufkirchen, Germany).

\section{Ion Mobility Experiments}

Experiments were performed in a prototype QTOF mass spectrometer maXis from Bruker Daltonik (Bremen, Germany). Compounds were dissolved in a (1:1) methanol-water solution with an addition of $1 \%$ formic acid. Sample concentrations in sprayed peptide sample solutions were at the level of $1-10 \mu \mathrm{g} / \mathrm{mL}$. In the mixtures of PEG 400 and prostaglandin $\mathrm{E}_{1}$ the sprayed concentrations were between $0.1-1 \mu \mathrm{g} / \mathrm{mL}$. Using the syringe pump infusion method, samples were sprayed with a flow rate of $3 \mu \mathrm{L} / \mathrm{min}$ into the electrospray ionization source. The angled sprayer (Figure 1) was at ground potential. Nitrogen was used as a drying gas at a temperature setting of $200{ }^{\circ} \mathrm{C}$. Ions generated at atmospheric pressure entered the mass spectrometer through a glass capillary with metallized ends. At the entrance the spray shield voltage was $-4000 \mathrm{~V}$ and the capillary entrance voltage was $-4500 \mathrm{~V}$. Beyond the glass capillary, ions entered a two-stage tapered stacked ring ion guide system (ion funnels 1 and 2). The pressure in the ion funnel 1 region was about $3 \mathrm{mbar}$, in the ion funnel 2 region it was about 0.5 mbar. Ions exiting ion funnel 2 through its conductance-limiting base plate entered a multipole ion guide in a pressure region of $10^{-4}$ mbar. Consequently, a steady gas flow existed through the orifice of the base of funnel 2 into the multipole ion guide. The ion mobility experiments reported in this work are performed in the ion funnel 2.
The peak-to-peak amplitude of the guiding RF field in funnel 2 was $400 \mathrm{~V}_{\mathrm{pp}}$. The electrostatic barrier was generated in funnel 2 by applying a repelling DC voltage (barrier voltage), in addition to the existing bias voltage of the ion funnel. In the experiments the barrier voltage was increased in $0.1 \mathrm{~V}$ steps. In the first method a repelling potential was exclusively applied to the base plate of funnel 2 (Figure 1). Since funnel 2 usually had a DC bias around $30 \mathrm{~V}$ in these experiments, the repelling potential at the base plate was selected to be above this bias voltage, and increased by using $0.1 \mathrm{~V}$ every $10 \mathrm{~s}$. Only the experiments with PEGs and the prostaglandin $E_{1}$ were performed using the first method. In this method, the electric field of the base plate is expected to partially penetrate into the entrance of the multipole. Furthermore, ions traveling from funnel 2 into the multipole experienced an increasing accelerating potential as the barrier voltage was increased. To avoid any possible changes in the ion transmission, a second method was developed in which the potential difference between the end of funnel 2 and the multipole was kept constant. In this method, the base plate and the last plate of funnel 2 were maintained at a constant voltage level whereas the voltages of the upstream ion guiding system (capillary exit voltage, funnel 1 voltages, and funnel 2 entrance voltage) were progressively lowered (Figure 1). Since the electrodes of the funnel 2 were connected to each other via a resistor chain, a voltage gradient existed along the axis of the funnel and also increased with increasing barrier voltage. The experiments to separate $\left[\mathrm{Arg}^{8}\right]-$ vasopressin and [des-Glu ${ }^{1}$ ]-LHRH, as well as all experiments to measure the ion mobilities of various

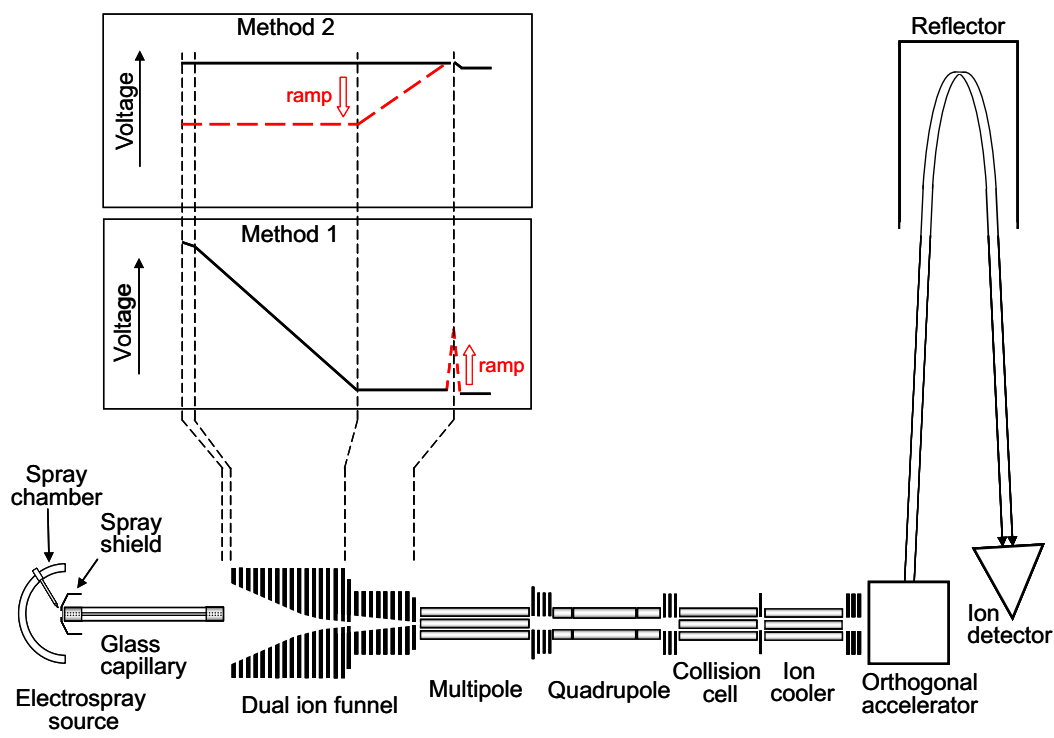

Figure 1. Schematic description of the two methods used for generating the potential barrier in the second ion funnel of the electrospray/mass spectrometer interface. In method 1, the barrier voltage is applied exclusively to the base plate of funnel 2, whereas in method 2, the base plate and the last plate voltages of funnel 2 were kept constant and the voltages of the upstream ion guiding system were progressively lowered. 
protonated peptides were performed using the second method.

The spray chamber (inner volume of approximately $1 / 3$ L) of the electrospray source was flooded with nitrogen as a drying gas. The drying gas temperature setting was $200^{\circ} \mathrm{C}$. Nitrogen entered the spray chamber between the front end of the electrospray capillary and the spray shield (Figure 1). In the experiments the flow rate of the drying gas was $4 \mathrm{~L} / \mathrm{min}$. When the sprayer operated the nebulizer gas-also nitrogen-entered the spray chamber with a flow rate of about $2 \mathrm{~L} / \mathrm{min}$. The nebulizer gas at ambient temperature, as well as the sprayed liquid that continuously evaporated, reduced the temperature in the chamber. Thus, the gas near the entrance of the electrospray capillary was at $\sim 120^{\circ} \mathrm{C}$. A more exact temperature measurement during the actual spray event was not possible due to spatial fluctuations of the temperature in the chamber. The solvent (roughly 50:50 methanol/water) flow rate out of the sprayer was $3 \mu \mathrm{L} / \mathrm{min}$, which would produce at an approximate temperature of $120^{\circ} \mathrm{C}$ roughly calculated $1.2 \mathrm{~mL} / \mathrm{min}$ of methanol vapor flow and $2.7 \mathrm{~mL} / \mathrm{min}$ water vapor flow. Therefore, during electrospray experiments the gas entering the electrospray capillary per unit time had a nitrogen:methanol:water composition of roughly 6000:1.2:2.7 in volume. The gas that entered the capillary and the ion funnels consisted over $99.9 \%$ of nitrogen.

The pressure in the ion funnel stages was much higher compared with pressures in the mass spectrometric vacuum. Thus, a frictional force caused by collisions in the gas flow dragged the ions out of the funnel into the mass spectrometer. To counteract the drag of the gas flow the electric field barrier was introduced and incrementally increased in the ion funnel. As the dragging effect of the gas flow was smaller for the more mobile ions, they could be blocked from entering the mass spectrometer by low barrier voltages. The influence of the gas flow on ions with lower mobility (larger cross sections, smaller number of charges) is stronger. They could be stopped by further elevating the barrier voltage. At a given point, only the ions with the lowest mobility could enter the mass spectrometer. A further voltage increase prevented all ions from passing through the orifice of the funnel base. The barrier voltage, at which a particular ion can be kept from entering the mass spectrometer, will be referred to as the blocking voltage in the following. It correlates to the mobility value of the corresponding ion. Ion mobility information can be obtained by ramping up the barrier voltage followed by plotting the ion signals with the characteristic signal intensity change at the blocking voltage and taking the derivative (slope) to represent the change of the signal as a peak. If the barrier voltage is increased the signal intensity change is a dropping curve at the blocking voltage. As its derivative is a negative peak, the negative derivative is used to obtain a positive peak. If the barrier voltage is scanned from high to low voltages, the signal intensity change is a rising curve, and its derivative is a positive peak. For those ions these peaks correspond to the blocking voltages which are, in turn, directly related to ion mobilities. Noisy or fluctuating ion signal plots lead to derivative curves that oscillate between positive and negative values. To reduce this unwanted effect, the signal plots are artificially "smoothed" using a method based on the Savitzky-Golay algorithm [23]. The DataAnalysis Software (Bruker Daltonik $\mathrm{GmbH}$, Bremen, Germany) used for the post-processing contains this smoothing function, as well as a tool for taking the derivative of the signal curve.

\section{Results}

\section{Selection by Ion Mobility}

Many drugs contain polyethylene glycols (PEGs) in addition to pharmaceutically active compounds. An experiment has been designed with prostaglandin $E_{1}$ to see how the polyethylene glycols PEG 400 and prostaglandin behave if the barrier voltage is increased. Polyethylene glycols have the following general formula:

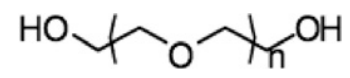

PEG 400 has, as the name indicates, a molecular weight distribution around 400. Using electrospray ionization sodium- (or potassium-) ion attached PEG molecules appeared as singly-charged ions. Prostaglandin $E_{1}$ has the following structure:

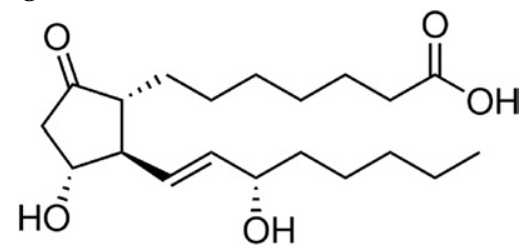

and an (average) molecular weight of 354.4. In the electrospray mass spectrum the monoisotopic signal of the sodiated prostaglandin $\mathrm{E}_{1}$ ion appears at $m / z$ 377.23. The results of first barrier voltage experiments showed that the PEGs around the sodiated prostaglandin ion disappeared at much lower voltages than the sodiated prostaglandin ion. In these experiments method 1 (Figure 1) was used. As the barrier voltage was increased, PEG peaks in the neighborhood of the prostaglandin peak were cleaned up in the mass spectrum. Prostaglandin's sodiated molecule was stopped at a slightly higher voltage than the sodiated PEG with $\mathrm{m} / \mathrm{z} 569$ (Figure 2a). As shown in Figure 2b, with the negative derivatives of the signal curves, the mobilities of the PEG ions of $\mathrm{m} / \mathrm{z} 305,349,393,437,481,525$, and 569 are all higher than the mobility of the sodiated prostaglan$\operatorname{din} \mathrm{E}_{1}$ at $\mathrm{m} / \mathrm{z}$ 377. Prostaglandin $\mathrm{E}_{1}$ does not have as many polar groups (hetero atoms) as polyethylene glycols, it has long side chains like wings, and with its ring it is less flexible than the PEGs: It cannot easily 


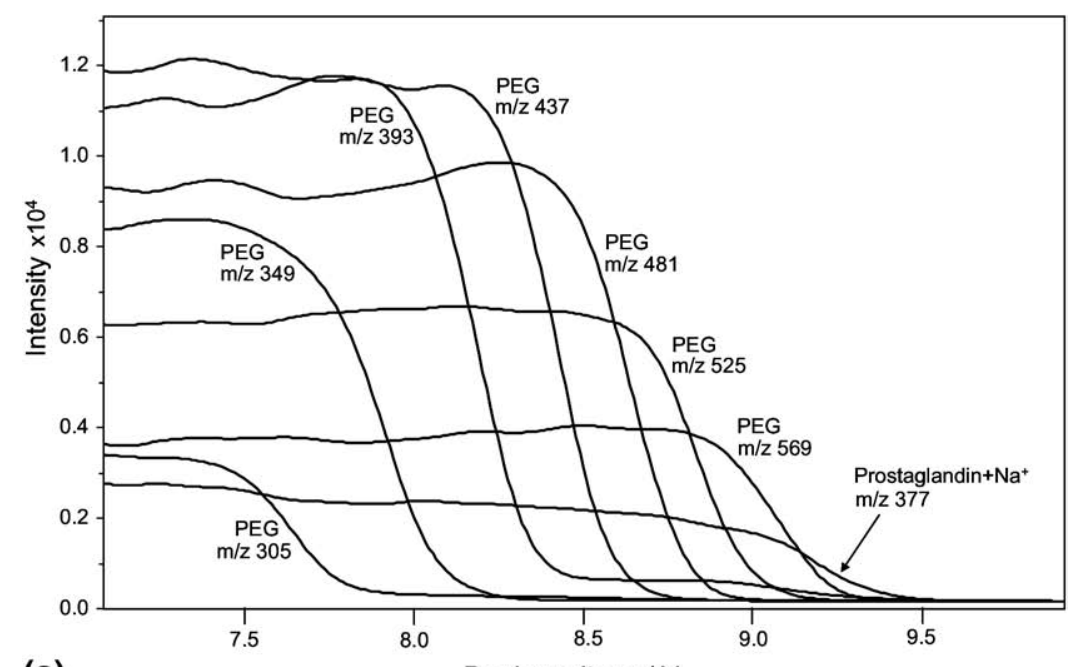

(a)

Barrier voltage / V

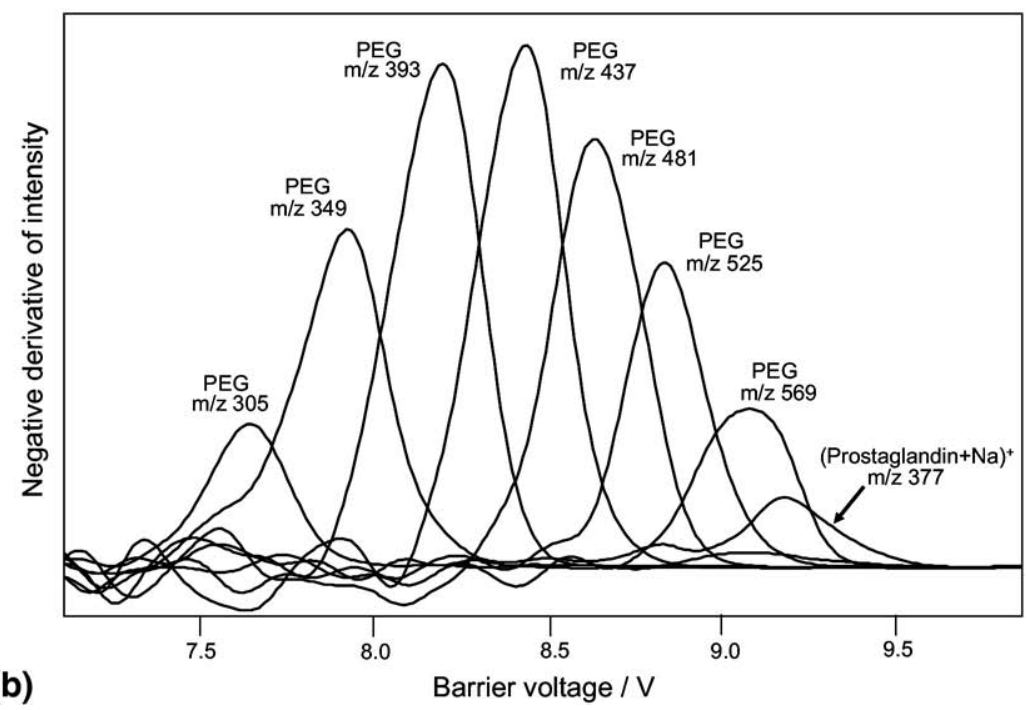

Figure 2. (a) Ion intensity plots of the sodium ion attached polyethylene glycols of PEG 400 and sodium ion attached prostaglandin $E_{1}$ during an increase of the voltage of the funnel 2 base according to method 1 ascertain the possibility of ion mobility-driven isolation. (b) Negative derivatives of the ion intensity plots shown in (a). In the derivative plot, the drop of the intensity curve is a negative peak, which is multiplied by -1 and plotted as a positive peak. The position of these peaks on the barrier voltage scale is related to the mobilities of the corresponding ions. Prostaglandin $\mathrm{E}_{1}$ has a lower mobility than the PEGs in the similar mass range. With increasing barrier voltage, all PEG peaks near prostaglandin disappear. Prostaglandin's signal intensity drops at relatively high barrier voltage values, almost at the same voltage as the PEG peak at $m / z 569$.

form folded structures. This makes its cross section larger than the PEGs of similar $m / z$, of which the sodiated molecules fold to more compact shapes. Ion mobility studies [24] show that the PEGs wrap around the sodium ion with its oxygen atoms binding to the ion analogous to crown ethers resulting in a very compact spherical structure. The mass spectra in Figure 3a clearly show that while at $6.5 \mathrm{~V}$ the sodium ion attached prostaglandin peak is surrounded by abundant PEG peaks, at elevated barrier voltages, the area near prostaglandin signal clears up. And at a barrier voltage of $9 \mathrm{~V}$ there were no PEG peaks over $\mathrm{m} / \mathrm{z} 500$ left. A two-dimensional signal display of mobilities versus $m / z$ of these ions is shown in Figure 3b. The PEG homologue series describe diagonal patterns in the display and the prostaglandin is singled out, as it belongs to an entirely different group of compounds.

Ions with Very Close m/z, Equal Charges, But Different Shapes

An experiment was designed with $\left[\mathrm{Arg}^{8}\right]-$ vasopressin (average MW: 1083.4): 

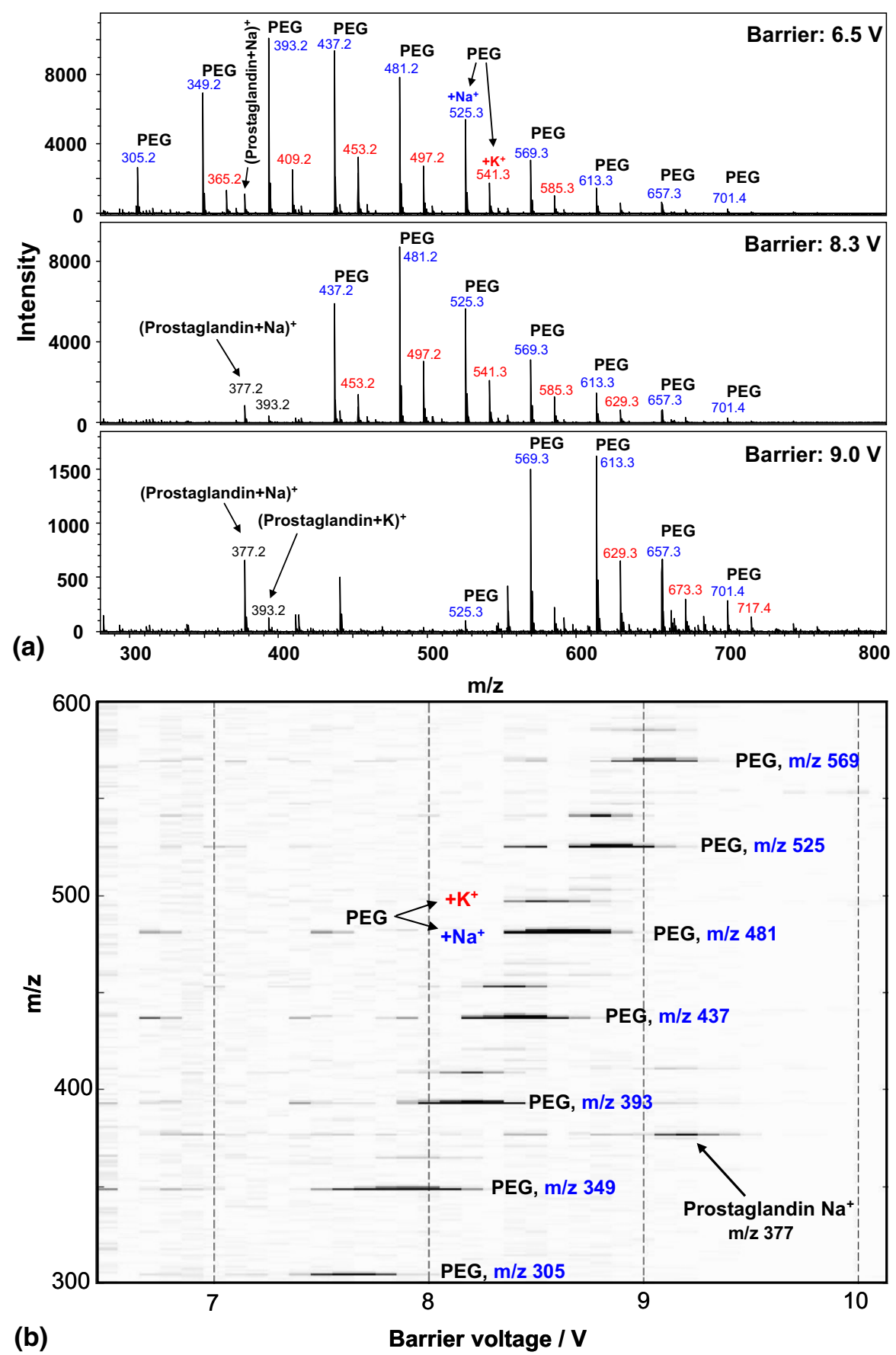

Figure 3. (a) Mass spectra of the prostaglandin $E_{1}+$ PEG 400 mixture at different barrier voltages. At $6.5 \mathrm{~V}$, the sodium ion attached prostaglandin and potassium ion attached prostaglandin peaks are surrounded by abundant peaks of sodium ion attached and potassium ion attached PEGs. In the displayed mass spectra, the mass numbers of sodiated PEGs are printed in blue, those of potassiated PEGs are in red. At $8.3 \mathrm{~V}$ the area near the prostaglandin peak is already cleared of the prostaglandins of similar mass-to-charge ratio. At a barrier voltage of $9 \mathrm{~V}$ the sodiated prostaglandin is almost singled out, and there are no PEG peaks in the spectrum left until over $m / z 500$. (b) A diagram of barrier voltages in relation of the mass-to-charge ratios in the mixture of PEG 400 and prostaglandin $\mathrm{E}_{1}$. The signal intensities in this diagram are shown by the grey tones so that the highest peaks are dark grey or black. This plot shows that the pattern of polyethylene glycols is entirely separated from that of prostaglandin $E_{1}$. Note that the main pattern of the sodium ion attached PEGs are accompanied by a secondary and weaker pattern of potassium ion attached PEGs. The repeating pattern of sodium and potassium attached PEGs are shown at an example peak (spot) pair. 


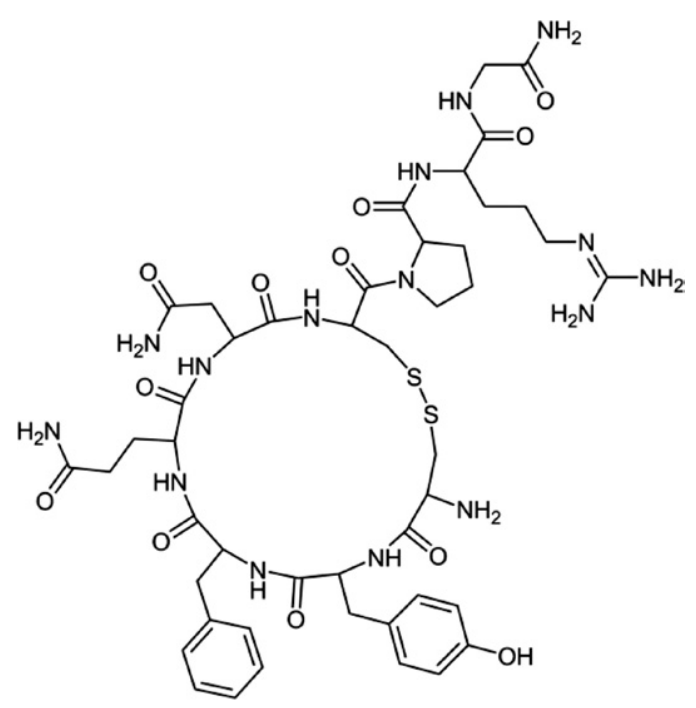

and [des-Glu ${ }^{1}$-LHRH with the sequence: His-Trp-SerTyr-Gly-Leu-Arg-Pro-Gly- $\mathrm{NH}_{2}$ (average $\mathrm{MW}$ 1071.19), which is a linear-chain peptide, to show effect of the cross section effects on the ion mobility. The comparison of doubly protonated $\left[\mathrm{Arg}^{8}\right]$-vasopressin (monoisotopic: $\mathrm{m} / \mathrm{z}$ 542.73) and doubly protonated [des$\mathrm{Glu}^{1}$ ]-LHRH (monoisotopic: $\mathrm{m} / \mathrm{z}$ 536.28) eliminates most of the mass and charge effects on the mobility, since the masses of these peptides are very close to each other, and the charges are the same when investigating the doubly protonated molecules. The only contribution to the mobility difference between these peptides would be by their cross sectional differences. Peptide chains of similar masses usually show similar compactness in structure. In this example, $\left[\mathrm{Arg}^{8}\right]$-vasopressin seems to be a more compact molecule due to the ring structure with its S-S bridge, while [des-Glu $\left.{ }^{1}\right]-$ LHRH is an open-chained peptide molecule. Figure 4 shows the negative derivatives of the intensity drop curves of $\left[\mathrm{Arg}^{8}\right]-$ vasopressin and [des-Glu $\left.{ }^{1}\right]-$ LHRH. Even though the mass of $\left[\mathrm{Arg}^{8}\right]$-vasopressin is slightly larger than the mass of [des-Glu $\left.{ }^{1}\right]-\mathrm{LHRH}$, the cross sectional difference between the two peptides predominates in the experiment. $\left[\mathrm{Arg}^{8}\right]$-vasopressin $+2 \mathrm{H}^{+}$ was blocked at $0.71 \mathrm{~V}$ lower barrier voltage than [des$\left.\mathrm{Glu}^{1}\right]-\mathrm{LHRH}+2 \mathrm{H}^{+}$. These relatively small and doubly protonated peptide chains most likely open up due to the Coulomb repulsion. The separation of the two charges and the associated increase of the cross section are apparently more successful in the linear [des$\left.\mathrm{Glu}^{1}\right]-\mathrm{LHRH}+2 \mathrm{H}^{+}$than in the $\left[\mathrm{Arg}^{8}\right]-$ vasopressin + $2 \mathrm{H}^{+}$ion which contains an S-S bridged ring structure.

\section{Ion Mobility Determination}

For quantitative information about ion mobilities, the barrier voltage scale has to be calibrated. A calibration can be carried out by using ions of known mobilities or cross sections in the same buffer gas as in our experiments. As described in the experimental section, the

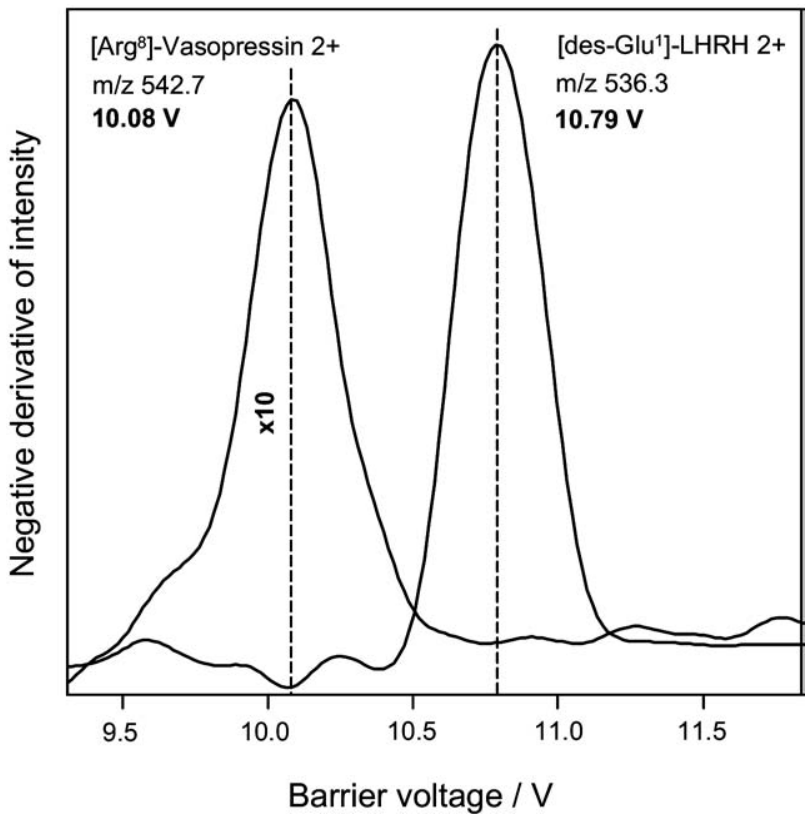

Figure 4. Negative derivatives of intensity plots of doublyprotonated [des-Glu $\left.{ }^{1}\right]-$ LHRH and doubly protonated $\left[\mathrm{Arg}^{8}\right]-$ vasopressin. $\left[\mathrm{Arg}^{8}\right]$-vasopressin $+2 \mathrm{H}^{+}$has a higher mobility than $\left[\right.$ des-Glu $\left.{ }^{1}\right]-\mathrm{LHRH}+2 \mathrm{H}^{+}$.

electrospray source is continuously flushed with nitrogen. Thus, the gas that entered the electrospray capillary and the ion funnels consisted of over $99.9 \%$ of nitrogen. Therefore, reference data from classic ion mobility experiments made in nitrogen buffer gas is used, since the cross sections are also collision gas dependent. Cross section information for ions of bradykinin, angiotensin I, fibrinopeptide A, and neurotensin in nitrogen were kindly provided by Dr. Erin S. Baker (Table 1). From these cross section values $(\sigma)$ ion mobilities K could be calculated [1] using the equation:

$$
K=\frac{3 z e}{16 N}\left(\frac{2 \pi}{\mu k_{B} T}\right)^{1 / 2} \frac{1}{\sigma}
$$

in which $z$ is the charge of the ion, $N$ is the number density, $k_{B}$ is the Boltzmann constant, $T$ the absolute temperature, and $\mu$ the reduced mass $\mu=m M /(m+M)$ with $m$ as the mass of the collision gas (nitrogen) molecule and $M$ as the mass of the analyte ion. Reduced mobilities $\mathrm{K}_{0}$ were obtained with the temperature $\mathrm{T}$ and pressure $\mathrm{p}$ correction:

$$
K_{0}=K \times \frac{T_{0}}{T} \times \frac{p}{p_{\text {atm }}}
$$

where $p_{a t m}$ is the atmospheric pressure 1013 mbar and $\mathrm{T}_{0}$ is $273.16 \mathrm{~K}$. In addition to the four reference peptides mentioned above, the penta-peptide Gly-Arg-Gly-AspSer (GRGDS) was also used. The singly protonated 
Table 1. Reference peptides used for calibration of the ion mobility spectra. Reduced mobility values are calculated back from cross section values using the experimental conditions

\begin{tabular}{lrrl}
\hline \multicolumn{1}{c}{ Peptide ion } & \multicolumn{1}{c}{$\begin{array}{c}\text { Cross } \\
\text { section } \\
\text { in } \mathrm{N}_{2}\left(\AA^{2}\right)\end{array}$} & $\begin{array}{l}\text { Reduced } \\
\text { mobility }\end{array}$ \\
\hline \hline Angiotensin $\mathrm{I}+3 \mathrm{~K}^{+}$ & 432.9 & $\left.47 \mathrm{~cm}^{2} \mathrm{~V}^{-1} \mathrm{~s}^{-1}\right)$ & 1.297 \\
Bradykinin $+2 \mathrm{H}^{+}$ & 530.8 & $332,340^{*}$ & $1.234,1.205$ \\
Fibrinopeptide $\mathrm{A}+2 \mathrm{H}^{+}$ & 768.9 & $401,413^{*}$ & $1.018,0.988$ \\
Fibrinopeptide $\mathrm{A}+3 \mathrm{H}^{+}$ & 513.3 & $481^{*}$ & 1.273 \\
Neurotensin $+3 \mathrm{H}^{+}$ & 558.3 & $494,522^{*}$ & $1.239,1,172$ \\
Gly-Arg-Gly-Asp-Ser $+\mathrm{H}^{+}$ & 491.2 & $185[7]$ & 0.85 \\
\hline
\end{tabular}

${ }^{*} \mathrm{~N}_{2}$ cross section values of these reference compounds were kindly provided by Erin S. Baker, Pacific Northwest National Laboratories.

molecule of GRGDS was a convenient example to generate a reference point for calibration in the lower mobility range [7]. Table 1 shows, for the reference peptides, the cross section data for nitrogen as buffer gas and the reduced mobilities calculated from these cross sections.

Figure 5a shows negative derivatives of ion intensities of the reference peptides versus the increased barrier voltage. The barrier voltage at every peak tip is marked as the blocking voltage for the corresponding compound. The cross section information about the ions of bradykinin, angiotensin I, neurotensin, and fibrinopeptide A was available from classic ion mobility mass spectrometry measurements in nitrogen buffer gas at 5.33 mbar pressure and $298 \mathrm{~K}$ temperature. $\mathrm{K}_{0}$ values were calculated using these conditions from the experiments in a classic drift tube. Only the experiments in reference [7] using the peptide GRGDS were performed at $523 \mathrm{~K}$. The corresponding $\mathrm{K}_{0}$ was also listed in the reference but also verified by calculation taking into account the specific experimental conditions. [Baker, E. S., Pacific Northwest National Laboratories, private communication on nitrogen cross section values of ions of bradykinin, angiotensin I, neurotensin, fibrinopeptide $\mathrm{A}$, measured by using classic drift tube ion mobility spectrometry at 4 Torr (5.33mbar) and $298 \mathrm{~K}]$.

A calibration curve between the reduced mobilities and the blocking voltages was plotted. Different cross section values were measured with classic ion mobility drift cells for three of the calibrant ions (bradykinin + $2 \mathrm{H}^{+}$, fibrinopeptide $\mathrm{A}+2 \mathrm{H}^{+}$, and neurotensin + $\left.3 \mathrm{H}^{+}\right)$. As these cross sections were very close to each other (Table 1), we used average values for our calibration. The calibration curve using the internal standards with known $\mathrm{K}_{0}$ is approximately a straight line in this range (Figure $5 b$ ). The two peptides [des-Glu ${ }^{1}$ ]-LHRH and $\left[\mathrm{Arg}^{8}\right]$-vasopressin (discussed above) with unknown mobilities were measured using the reference peptides as internal standards. Additionally, mobilities could also be determined for doubly protonated angiotensin I and doubly protonated neurotensin, for which the cross section reference data were not available.
The $K_{0}$ values from these experiments showed a linear dependence (with a negative coefficient) on the blocking voltages of measured reference peptides. If the ionic cross section values were picked for calibration purposes instead of the $\mathrm{K}_{0}$, for ions of each charge state a separate calibration curve would be necessary. To have a single calibration curve for ions the "shape-tocharge" ratio $(\sigma / \mathrm{z})$ values should be plotted versus the blocking voltage. In this case, all points are part of the same calibration curve, but this curve did not show a linear dependence on the blocking voltage. Therefore, in this range of mobilities, we chose to calibrate with the $\mathrm{K}_{0}$ values and then calculated the $\sigma$ values of all tested compounds by considering again the experimental conditions in classic IMS experiments initially defined for the reference compounds (5.33 mbar and $298 \mathrm{~K}$ ).

In a further experiment, the ion mobilities of the common peptides angiotensin II, substance $\mathrm{P}$, bombesin, and melittin were measured. As internal calibrants the same reference peptides as above were used. Figure $6 \mathrm{a}$ shows negative derivatives of ion intensities versus the increased barrier voltage in this experiment. The barrier voltage at every peak tip is, again, marked as the blocking voltage for the corresponding compound. Using the calibration line the $\mathrm{K}_{0}$ values could be determined for doubly protonated molecules of angiotensin II, substance $\mathrm{P}$, and bombesin, as well as for triply and quadruply protonated molecules of melittin (Figure $6 b$ ). A list of the determined mobilities of the peptides used in the two experiments above is shown in Table 2.

\section{Discussion}

The experiments show that ion mobility can be measured in an ion funnel interface of an electrosprayorthogonal time-of-flight mass spectrometer by introducing a barrier voltage and ramping it. An internal calibration with compounds of known ion mobility or cross section enables the ion mobility of compounds under investigation to be determined.

Using this dynamic method, mobility resolving powers up to 30 were observed with the measured compounds. A set of routinely obtained mobility resolving power $\mathrm{R}_{\mathrm{m}}=\mathrm{K}_{0} / \Delta \mathrm{K}_{0}$ values observed at various peaks were (FWHH) $R_{m}=30$ at angiotensin II $2+, R_{m}=25$ at fibrinopeptide A $3+, R_{m}=25$ at angiotensin $3+, R_{m}=$ 21 at bombesin, $R_{m}=25$ at melittin $4+$, and $R_{m}=16$ at melittin $3+$. Since the signal intensity curve needs initially to be smoothed before the derivative is taken, parameters involved in this smoothing [23] operation are selected in a way to eliminate the noise-related intensity fluctuations but not a peak structure arising from actual physical differences in ions. Smoothing widens the mobility peaks a little and therefore slightly reduces the actual resolving power in the ion mobility spectrum.

The experiments we presented also showed that ion mobility-related filtering is not only useful for filtering the low mass ions [22], but it can also be used as a more 
(a)
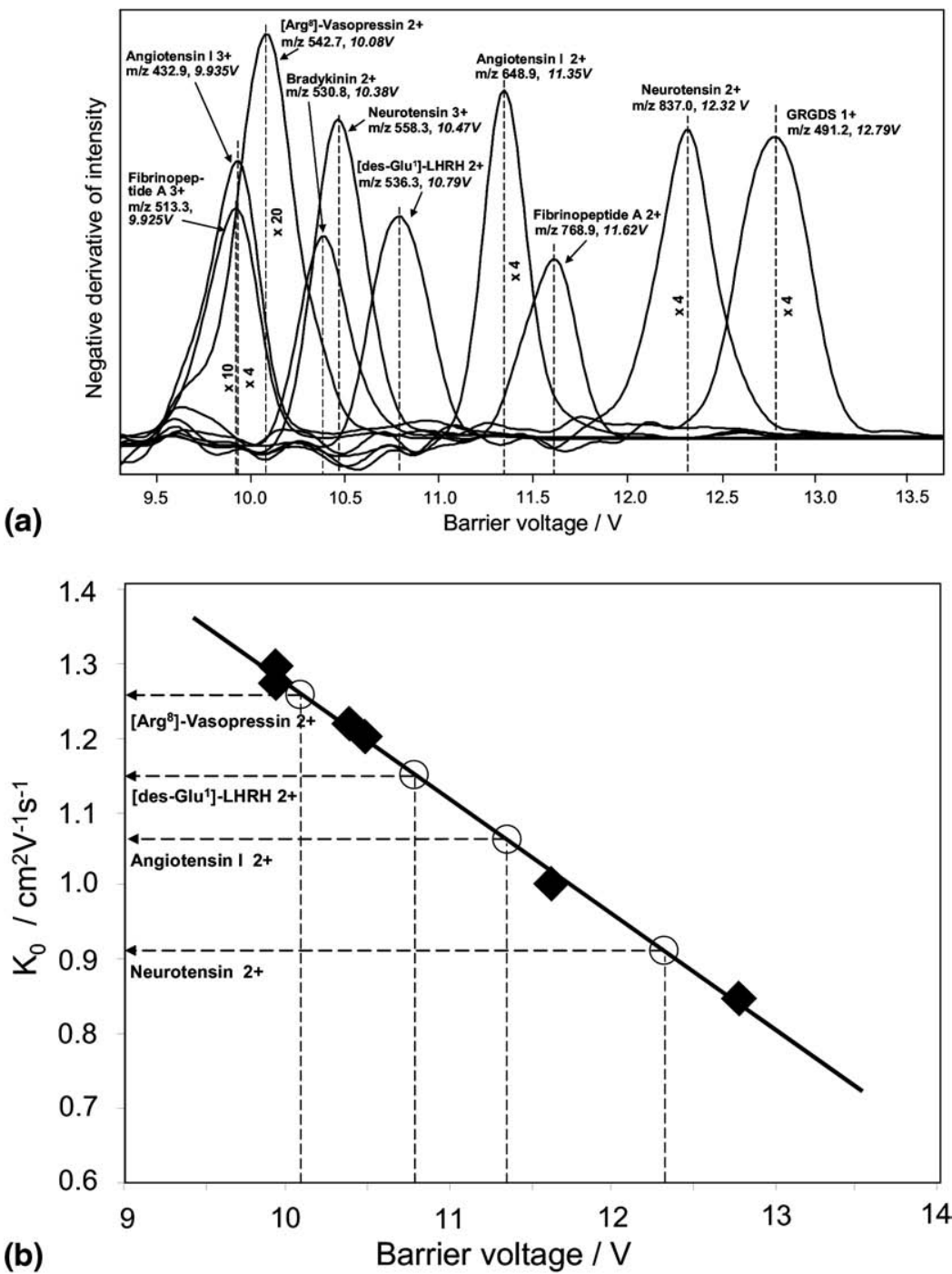

Figure 5. (a) Negative derivatives of intensity plots of various peptide ions to determine ion mobilities. In this experiment, the internal calibrants were angiotensin $\mathrm{I}+3 \mathrm{H}^{+}$, bradykinin $+2 \mathrm{H}^{+}$, fibrinopeptide $\mathrm{A}+2 \mathrm{H}^{+}$, fibrinopeptide $\mathrm{A}+3 \mathrm{H}^{+}$, neurotensin $+3 \mathrm{H}^{+}$, and Gly-Arg-Gly-Asp-Ser + $\mathrm{H}^{+}$. In order not to complicate the diagram, only the charge states of the ions are indicated in the ion mobility spectrum, and not the protons. (b) Calibration curve and the determination of mobilities of doubly-protonated [des-Glu $\left.{ }^{1}\right]-\mathrm{LHRH}$ and $\left[\mathrm{Arg}^{8}\right]-$ vasopressin. Diamonds indicate the internal calibrant points angiotensin $\mathrm{I}+3 \mathrm{H}^{+}$, bradykinin $+2 \mathrm{H}^{+}$, fibrinopeptide $\mathrm{A}+2 \mathrm{H}^{+}$, fibrinopeptide $\mathrm{A}+$ $3 \mathrm{H}^{+}$, neurotensin $+3 \mathrm{H}^{+}$, and Gly-Arg-Gly-Asp-Ser $+\mathrm{H}^{+}$[see in (a)]. The mobilities of angiotensin $\mathrm{I}+2 \mathrm{H}^{+}$, and neurotensin $+2 \mathrm{H}^{+}$, which were also generated in the ionization process of the internal calibrants (reference list, Table 1) have also been determined on this occasion.

general way of filtering if a less mobile analyte is contaminated with more mobile compounds (e.g., additives) in the same mass range. This way, it is possible to clean-up the signals of more mobile compounds from the neighborhood of an analyte peak without a mass preselection (without MS/MS). Experiments with prostaglandin $\mathrm{E}_{1}$ and PEG 400 are a prime example for this application.

During the ion mobility measurements, all changes made to the normal operation of the hardware were kept to a minimum. The electrospray ion source was, for example, continuously operating and producing ions while the barrier voltage was being ramped. The elevated barrier voltage may lead to an increase of the analyte ion population in the second ion funnel compared to a barrier-free flow.

\section{Conclusions}

The modified operational mode of the ion funnel selected for mobility experiments allowed the determination of mobilities of ions generated from a series of compounds. This turns out to be a convenient technique, since it utilizes the existing hardware of a Q-TOF mass spectrometer. Resolving powers obtained with this method for ion mobility are in the range between 20 
(a)
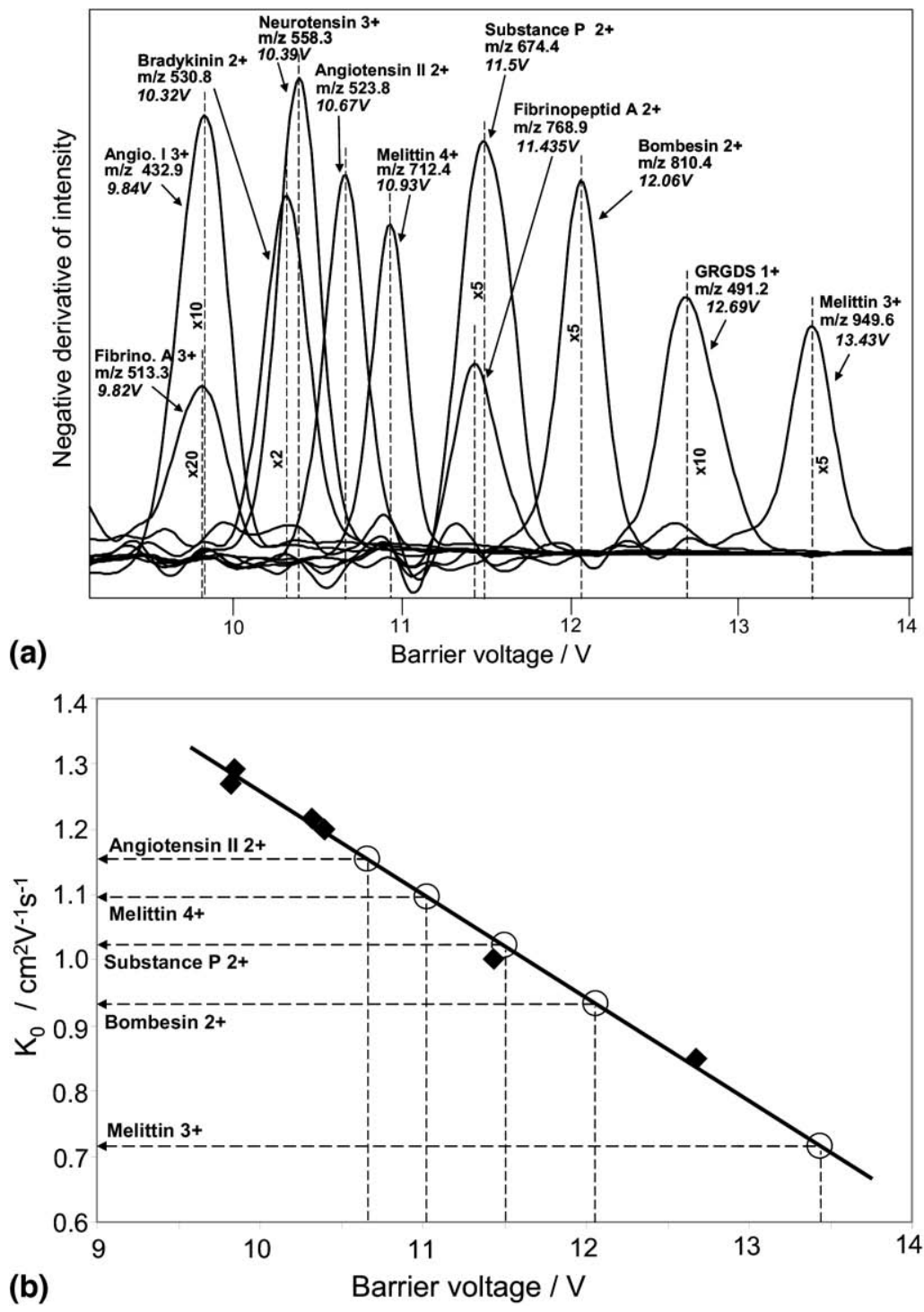

Figure 6. (a) Negative derivatives of intensity plots of various peptide ions to determine ion mobilities of ions generated from substance $\mathrm{P}$, angiotensin II, bombesin, and melittin. In this experiment the internal calibrants were angiotensin $\mathrm{I}+3 \mathrm{H}^{+}$, bradykinin $+2 \mathrm{H}^{+}$, fibrinopeptide $\mathrm{A}+$ $2 \mathrm{H}^{+}$, fibrinopeptide $\mathrm{A}+3 \mathrm{H}^{+}$, neurotensin $+3 \mathrm{H}^{+}$, and Gly-Arg-Gly-Asp-Ser $+\mathrm{H}^{+}$. In order not to complicate the diagram, only the charge states of the ions are indicated in the ion mobility spectrum, and not the protons. (b) The calibration curve and the determination of mobilities of doublyprotonated angiotensin II, bombesin, as well as triply and quadruply protonated melittin. Diamonds indicate the internal calibrant points angiotensin $\mathrm{I}+3 \mathrm{H}^{+}$, bradykinin $+2 \mathrm{H}^{+}$, fibrinopeptide $\mathrm{A}+2 \mathrm{H}^{+}$, fibrinopeptide $\mathrm{A}+3 \mathrm{H}^{+}$, neurotensin $+3 \mathrm{H}^{+}$, and Gly-Arg-Gly-Asp-Ser $+\mathrm{H}^{+}$[see in (a)].

and 30. Our experience shows that an internal calibration using compounds of known mobility in nitrogen is the safest way to assign the correct ion mobilities, as this assures that the conditions in the ion funnel are exactly the same. However, external calibrations also gave useful results when ion source, ion transfer, and sample conditions were not varied. Two-dimensional displays of measured ion mobility peaks (derivatives of ion signals) versus the $\mathrm{m} / \mathrm{z}$ of the measured ions represent a convenient way of giving an overview to the substance classes, as shown in the case with the PEG 400 and the prostaglandin $E_{1}$. The examples can be extended, and substance classes with generally compact structures will be separately displayed from those which have larger and extended structures.

In the way that the experiments are currently performed, a physical separation of compounds is limited. At a high barrier voltage, ions with the lowest mobility are the only ones to pass the funnel base plate and to enter the mass spectrometer. Thus, these ions are physically separated from the rest. However, if the barrier voltage is dropped, ions with higher mobilities also start passing the funnel base. So, instead of being a "moving window" allowing one mobility at a time to enter the mass spectrometer, this method is rather like a "curtain", which opens up from the lower end of the 
Table 2. Ion mobilities determined from internally calibrated barrier voltage plots. Corresponding ionic cross section values are calculated from the mobility data

\begin{tabular}{lccc}
\hline \multicolumn{1}{c}{ Peptide ion } & $\mathrm{m} / \mathrm{z}$ & $\begin{array}{c}\text { Reduced } \\
\text { mobility } \\
\mathrm{K}_{0}\left(\mathrm{~cm}^{2} \mathrm{~V}^{-1} \mathrm{~s}^{-1}\right) \\
\text { by calibration }\end{array}$ & $\begin{array}{c}\text { Calculated } \\
\text { cross } \\
\text { section in } \\
\mathrm{N}_{2}\left(\AA^{2}\right)\end{array}$ \\
\hline \hline Angiotensin I $+2 \mathrm{H}^{+}$ & 648.9 & 1.065 & 384 \\
Neurotensin $+2 \mathrm{H}^{+}$ & 837.0 & 0.912 & 447 \\
[Arg $^{8}$ ]-Vasopressin $+2 \mathrm{H}^{+}$ & 542.7 & 1.259 & 326 \\
[Des-Glu $^{1}$ ]-LHRH $+2 \mathrm{H}^{+}$ & 536.3 & 1.152 & 356 \\
Angiotensin II $+2 \mathrm{H}^{+}$ & 523.8 & 1.155 & 355 \\
Substance $P+2 \mathrm{H}^{+}$ & 674.4 & 1.022 & 400 \\
Bombesin $+2 \mathrm{H}^{+}$ & 810.4 & 0.932 & 438 \\
Melittin $+3 \mathrm{H}^{+}$ & 949.6 & 0.717 & 850 \\
Melittin $+4 \mathrm{H}^{+}$ & 712.4 & 1.097 & 741 \\
\hline
\end{tabular}

mobility spectrum. When the curtain is fully open, all ions enter the mass spectrometer.

At higher barrier voltages, more mobile ions are blocked, and since the electrospray source continues to produce ions, the total number of ions can increase in the second ion funnel. However, Coulombic repulsion in the dense ion cloud may lead to some losses and therefore limit the ion number. Yet, space charge effects are expected during the experiments. A study of these effects and their influence on the conducted ion mobility experiments has not yet been performed.

Other experiments can be envisioned in which a predefined ensemble of ions is generated and trapped using a high barrier voltage and gradually released by progressively decreasing the barrier. During this process, no new ions would be added to the ensemble, [Julian, R. R., University of California Riverside, private communication]. These experiments would allow a separation of ions in the trapped ensemble with regard to their mobility: Initially, the ions with lowest mobility would exit the trap, and later the ones with higher mobility would follow, and so on. Since the trapped ion ensemble would have only a limited number of ions, the sensitivity issue should be carefully examined in this case.

A quick comparison of the method we used with the experiments for determination of collision cross sections in the collision cell of tandem quadrupole mass spectrometers [17-19] shows the following: In the stationary gas of the collision cell the ions with smallest cross sections pass the stopping potential (a low pass filter in terms of cross sections) and are detected, while the collisions with ions of larger cross sections reduce their translational energy so that they cannot reach the analyzer quadrupole. However, in our experiments, where the ions are transported by the gas flow, ions with the largest cross sections pass the barrier (high pass filter) and are detected. A serial combination of these two techniques (high pass and low pass filters) could possibly enable an operation like a band-pass filter for selection of cross sections, i.e., mobilities.
In the current state of the technique, $0.1 \mathrm{~V}$ increments are made every $6 \mathrm{~s}$. Therefore, the experiments are not compatible with methods requiring faster response, e.g., a preseparation by liquid chromatography. Part of our current work concentrates on various modifications of this technique to enable a considerably faster barrier voltage scan.

\section{Acknowledgments}

The authors especially thank Dr. Erin Shammel Baker for the information on absolute cross sections of angiotensin I $3+$, fibrinopeptide A $2+$, and fibrinopeptide A $3+$, neurotensin $3+$ measured using classic ion mobility-mass spectrometry with nitrogen as a buffer gas. The authors' appreciation also goes to Professor Ryan Julian for initial discussions and suggestions on ion mobility measurements using a barrier voltage in an orthogonalacceleration time-of-flight mass spectrometer. The authors also thank Dr. Melvin Park, Dr. Ruediger Frey, Dr. Armin Holle, Dr. Ian Sanders, and Dr. Michael Schubert for helpful discussions.

\section{References}

1. McDaniel, E. W.; Mason, E. A. The Mobility and Diffusion of Ions in Gases. John Wiley and Sons: New York, 1973; pp 29-84, 118-235.

2. Viehland, L. E.; Mason, E. A. Gaseous Ion Mobility in Electric Fields of Arbitrary Strength. Ann. Phys. 1975, 91, 499-533.

3. Young, C. E.; Edelson, D.; Falconer, W. E. Water Cluster Ions: Rates of Formation and Decomposition of Hydrates of the Hydronium Ion. J. Chem. Phys. 1970, 53, 4295-4302 (also see Reference 1 pp. 69).

4. Clemmer, D. E.; Jarrold, M. F. Ion Mobility Measurements and Their Applications to Clusters and Biomolecules. J. Mass Spectrom. 1997, 92, 577-592.

5. Wyttenbach, T.; Kemper, P. R.; Bowers, M. T. Design of a New Electrospray Ion Mobility Mass Spectrometer. Int. J. Mass Spectrom. 2001, 212, 13-23.

6. Gidden, J.; Bowers, M. T. Gas Phase Conformations of Deprotonated and Protonated Mononucleotides Determined by Ion Mobility and Theoretical Modeling. J. Phys. Chem. B 2003, 107, 12829-12837.

7. Wu, C.; Siems, W. F.; Klasmeier, J.; Hill, H. H. Separation of Isomeric Peptides Using Electrospray Ionization/High Resolution Ion Mobility Spectrometry. Anal. Chem. 2000, 72, 391-395.

8. Dwivedi, P.; Wu, C.; Matz, L. M.; Clowers, B. H.; Siems, W. F.; Hill, H. Gas Phase Chiral Separations by Ion Mobility Spectrometry. Anal. Chem. 2006, 78, 8200-8206.

9. Gillig, K. J.; Ruotolo B. T.; Stone, E. G.; Russell, D. H.; Fuhrer, K.; Gonin, M.; Schultz, J. A. Coupling High-Pressure MALDI with Ion Mobility/Orthogonal Time-of-Flight Mass Spectrometry. Anal. Chem. 2000, 72, 3965-3971.

10. Ruotolo, B. T.; Gillig, K. J.; Woods, A. S.; Egan, T. F.; Ugarov, M. V. Schultz, J. A.; Russell, D. H. Analysis of Phosphorylated Peptides by Ion Mobility-Mass Spectrometry. Anal. Chem. 2004, 76, 6727-6733.

11. Woods, A. S.; Ugarov, M.; Egan, T.; Koomen, J.; Gillig, K. J.; Fuhrer, K.; Gonin, M.; Schultz, J. A. Lipid/Peptide/Nucleotide Separation with MALDI-Ion Mobility-TOF MS. Anal. Chem. 2004, 76, 2187-2195.

12. Baker, E. S.; Bernstein, S. L.; Bowers, M. T. Structural Characterization of G-Quadruplexes in Deoxyguanosine Clusters Using Ion Mobility Mass Spectrometry. J. Am. Soc. Mass Spectrom. 2005, 16, 989-997.

13. Bernstein, S. L.; Wyttenbach, T.; Baumketner, A.; Shea, J.E.; Bitan, G.; Teplow, D. B.; Bowers, M. T. Amyloid $\beta$-Protein: Monomer Structure and Early Aggregation States of A $\beta 42$ and its Pro ${ }^{19}$ Alloform. J. Am. Chem. Soc. 2005, 127, 2075-2084.

14. Dupuis, N.; Kemper, P.; Bowers, M. T. Design and Operation of a New High Resolution Ion Mobility Mass Spectrometer. Proceedings of the ASMS Conference on Mass Spectrometry and Allied Topics; Denver, CO, June, 2008.

15. Baker, E. S.; Tang, K.; Danielson, W. F. III; Prior, D. C.; Smith, R. D. Simultaneous Fragmentation of Multiple Ions Using IMS Drift Time Dependent Collision Energies. J. Am. Soc. Mass Spectrom. 2008, 19, 411-419.

16. Tang, K.; Shvartsburg, A. A.; Lee, H.N.; Prior, D. C.; Buschbach, M. A Li, F.; Tolmachev, A. V.; Anderson, G. A.; Smith, R. D. High-Sensitivity Ion Mobility Spectrometry/Mass Spectrometry Using Electrodynamic Ion Funnel Interfaces. Anal. Chem. 2005, 77, 3330-3339.

17. Covey, T.; Douglas, D. J. Collision Cross Sections for Protein Ions. J. Am. Soc. Mass Spectrom. 1993, 4, 616-623.

18. Cox, K. A.; Julian, R. K. Jr.; Cooks, R. G. Conformer Selection of Protein Ions by Ion Mobility in a Triple Quadrupole Mass Spectrometer. J. Am. Soc. Mass Spectrom. 1994, 5, 127-136. 
19. Gill, A. C.; Jennings, K. R.; Wyttenbach, T.; Bowers, M. T. Int. J. Mass Spectrom. 2000, 195/196, 685-697.

20. Fernandez-De La Mora, J.; Ude, S.; Thomson, B. A. The Potential of Differential Mobility Analysis Coupled to MS for the Study of Very Large Singly and Multiply Charged Proteins and Protein Complexes in the Gas Phase. Biotechnol. J. 2006, 1, 988-997.

21. Kim, T.; Tolmachev, A. V.; Harkewicz, R.; Prior, D. C.; Anderson, G. Udseth, H. R.; Smith, R. D.; Bailey, T. H.; Rakov, S.; Futrell, J. H. Design and Implementation of a new Electrodynamic Ion Funnel. Anal. Chem. 2000, 72, 2247-2255
22. Page, J. S.; Tolmachev, A. V.; Tang, K.; Smith, R. D. Variable Low Mass Filtering Using an Electrodynamic Ion Funnel. J. Mass Spectrom. 2005, 40, 1215-1222.

23. Savitzky, A.; Golay, M. J. E. Smoothing and Differentiation of Data by Simplified Least Squares Procedures. Anal. Chem. 1964, 36, 16271639.

24. von Helden, G.; Wyttenbach, T.; Bowers, M. T. Inclusion of a MALDI Ion Source in the Ion Chromatography Technique: Conformational Information on Polymer and Biomolecular Ions. Int. J. Mass Spectrom. Ion Processes 1995, 146/147, 349-364. 